\title{
Metacognitive skills training effect on cognitive function in (TBI) patients. A Systematic Review
}

\author{
Sara A Makka \\ Intern Neuropsychologist, Intern Clinical Psychologist, Beirut, Lebanon
}

\begin{abstract}
Metacognition is among the current controversial neuropsychological topics which-had its influence on cognition in ill and healthy individuals. Many cognitive failures that (TBI) patients endure are not tackled in standardized protocols of cognitive rehabilitation efforts. One of the debilitating features of (TBI) is the lack of self-awareness of the disease. Metacognitive training can help in diminishing rehabilitation resisting obstacles. Not many studies to our knowledge have been done on meta-cognitive rehabilitation efforts in the case of (TBI) patients. In this review, electronic database is searched in strict inclusion exclusion criteria, eight studies have been included in a descriptive analysis. This might determine the power of evidence on the effect of metacognitive skills and strategy training on cognitive function in (TBI) patients. Studies recruited ranged from addressing executive function, social skills, to general cognitive competences. All of the studies proved the significance of metacognitive interventions in altering cognitive rehabilitation outcome but more attention should be given to the generalization of learned skills to daily life.
\end{abstract}

\section{Introduction and Literature}

(TBI) can influence several levels of cognitive function depending on the severity and site of the injury. One of the most weakening consequences is the unawareness of the patient's error or the illness itself. This stands out as a great hinderer for rehabilitation efforts. The knowledge of someone's circumstances can be referred to as metaknowledge. Metacognition is similarly the cognition of cognition or, if put simple, the ability to introspect personal thought and cognition. Metacognitive interventions aim to elevate the patient's awareness of his situation and the surrounding and teach him better strategies to address his fallibilities. The main point of strength that arms the injured patient with during metacognitive training is realizing his errors and increasing his capacity to auto-correct them. If metacognitive interventions proved successful, many rehabilitation resistance can be decreased by raising the self-awareness and insight in the patient's eyes. In addition to raising the efficacy of rehabilitation, many caregivers and family members will find better guided ways to deal with the patient as they better understand the "denial of state" they accuse the patient to produce. Metacognitive training can also address other functional domains of cognition that can be later on investigated. Literature has been conducted on the efficacy of metacognitive training or other approaches which have been formulated based on metacognitive principles and have proved themselves significant. With that mentioned, metacognitive training doesn't seem to be the most spontaneous and direct preliminary step done when conducting psychometric assessment for post hospitalization care. To better assess where metacognitive training and strategy training has evolved and hence decide whether cognitive rehabilitation and (TBI) assessment protocol classification steps should be reconsidered, evidence should be accumulated and collected. This will help clinicians include metacognitive training as an initial pre/post assessment outcome and a protocol in neuro-rehabilitation and cognitive rehabilitation.

\section{Objective}

This review aimed to determine the effect of metacognitive skills training on global and secondary cognitive function in Traumatic Brain Injury Patients.

\section{Methods}

Using the "Preferred Reporting Items for Systematic Reviews and Meta-Analyses" (PRISMA) 2009 guidelines, one researcher independently performed the search using two database namely; National Library of Medicine (Pubmed) and Cochrane Library.

\section{Criteria for considering studies for this review}

\section{Types of studies}

Randomized Clinical Trials were included in addition to single and series case studies which had an experimental design.

\section{Types of participants}

Pediatric and adult participants suffering from TBI (mild, moderate, to severe) were recruited. Studies were selected where participants were matched. Participants with head injuries that were not considered as TBI were excluded.

\section{Types of interventions}

Metacognitive intervention as an intervention: Interventions that are specifically targeted at restoring components of metacognition or any of its relevant aspect such as self-awareness or mediated learning were included. In this sense, if metacognitive skills training was performed in combination with another technique that would be by definitions also metacognitive, the study would be included. However, if the metacognitive intervention was used in combination with another

${ }^{\star}$ Correspondence to: Sara A Makka, Intern Neuropsychologist, Intern Clinical Psychologist, Beirut, Lebanon, Tel: +96176706843; E-mail: saramakke96@ gmail.com

Key words: metacognition, (TBI), cognitive outcome, cognition, metacognitive skills training

Received: October 18, 2020; Accepted: November 10, 2020; Published: November 13, 2020 
intervention that was not encompassed under metacognition then the study wouldn't have been recruited.

Metacognitive intervention as a comparison control: Metacognitive interventions in the systematic review performed in the presented study intend to study the effect of (MST) as the direct cognition effector but many studies recruited used (MST) as a control intervention and hence were not recruited.

An intervention with Metacognitive objectives: It must be noted that some metacognitive interventions would not be in abstract or keywords referred to as "metacognitive" intervention but as "mediated learning" or "error awareness" or any other naming but will be representing a module within metacognitive components of intervention and will be asserted in the full text of study as a metacognitive intervention.

\section{Types of Outcome Measures: global cognitive function or components within cognitive function}

The gold standard of outcome measurement is pre- versus post assessment differential in functional status [1].

The primary outcome is global cognitive function, and the secondary outcomes are components of cognitive function but, as most studies which address cognitive function as a global primary outcome would be having cognitive rehabilitation as an effector instead of (MST). For this cause, any component of cognition or a component of cognition compartments studied was considered. This includes: social competences, executive function, error detection, discourse and speech, etc.,

\section{Results}

\section{Electronic Search Results}

The electronic searches yielded 393 records and, after the initial screening of titles and abstracts by the review author (SM), (376) irrelevant papers were eliminated. (21) studies were to be included according to the pre-set inclusion criteria but (11) were excluded for a total of (8) studies to be finally eligible for the descriptive analysis.

Thus, 8 studies were eligible for inclusion (71 participants) in this review [2-10].

\section{Discussion and Description of Results}

\section{Summary of main results}

Out of the 8 studies included, studies related to four key comparisons: Metacognitive interventions targeting error awareness and self-correction (2 studies) [8,9]. Metacognitive interventions targeting executive function (3 studies) $[3,6,10]$. Metacognitive interventions targeting social competences (2 studies) [2] and Metacognitive intervention targeting global cognitive function with focus on problem solving in one study [11]. All studies proved efficacy and significance of their proposed interventions as they have reported.

\section{Significance of Studies}

Metacognitive interventions targeting error awareness and selfcorrection: It is good to note that executive functioning encompasses error detection and self-regulation and hence interventions which as stated above targeting self-regulation can be considered in the same set [12] yet and after reviewing literature and due to the specificity of self-awareness and regulation debilitation as a major concern in TBI, it has been reconsidered as a category on its own especially that studies targeting executive function addressed it as a global executive function and not branched or specified. The two studies $[8,9]$ were conducted by the same first author and aimed to apply metacognitive skills training sessions in comparison to other behavioural interventions and used appropriate instrument to measure outcome and monitor psychological functioning alongside. Both studies showed significant decrease in error frequency and a significant increase in error detection which shows that (MST) can have a positive effect on error awareness in TBI patients. To better appreciate the importance of (MST) in error awareness, one must know that poor awareness of behavior, emotions, and disability had been linked to less rehabilitation efficacy and more resistance. Better awareness of mistakes and errors would practically decrease forgetting to lock the doors for example which will by product increase the patient's independence and decrease the need and worry of the caregiver to stay around [13]. Gaining better cognitive control of consciousness will help the patient enhance his sustained attention versus distractibility that he usually suffers from [14].

Metacognitive interventions targeting executive function: A successful holistic rehabilitation program aims to address the ability to generalize skills remediated at the center to the daily life of the patient. This ability to generalize and extend the mediated learning of skills is better enhanced by cognitive flexibility that is one of the components of executive function [12,15]. In those studies, generalization was addressed. Group analysis showed a significant improvement in executive function and the investigation of the efficacy of the Cognitive Orientation to daily Occupational Performance (COOP) approach also showed significant results for the extension of the learned skills to the daily life activities $[3,6,10]$. The third study though used Goal Management Training but showed union of intersection with Metacognitive Skills Intervention and has also extended its area of investigation to detect whether those generalized concepts can extend to long time after the injury [10]. Unfortunately, this was not achieved and the self-informant questionnaires showed that the skills couldn't be generalized from the clinic/center to daily life activities. The study itself recommended further improvements and elaborations on this level even though (GMT) proved itself efficacious in the rehabilitation of combat veterans specifically in the executive function aspect. Transfer and generalization of strategy is essential in any executive function enhancement regime [16] because it is not enough to regain skills strictly in clinic. Add to that, our perception and view of learning shouldn't be solely depending on the learned subject. If a clinician is trying to address a certain skill (cooking a meal for example), planning as conveyed by neural behavior is not restricted to planning the steps to cooking the meal only, instead, it is a generalized gained skill that will affect all "To-be-planned" subjects. Insight this, if generalization fails to be significant then the intervention might (and might not be) successful in clinical contexts only - one of the problems caregivers and family members argue about- because it is of major clinical importance to maintain treatment [17]. Clinicians are not only interested in documenting impairment but are also interested in developing functional goals of therapy.

Metacognitive interventions targeting social competences: Social communication was addressed in two studies [2,4]. Yet, in the Remediation of Social Communication Study, the metacognitive intervention targeted social communication but the outcome of social communication was assessed by linguistic measures which divert the focus from a social context to a language discourse one (Profile of Pragmatic Impairment in Communication (PPIC) was 
used in addition to other instruments). Nonetheless, discourse is an essential component in social skills and communication especially that cognitive functional domains include language and cannot be perceived as unique circuits each serving the function on its own [18]. The second study was a modified intervention that was originally created to rehabilitate schizophrenic patients and have been divided to eight interesting modules that do not solely focus on social skills or communication [2]. All studies showed significant results. It is good to mention that even though social communication is a very prominent feature in many brain injury conditions as well as neuropsychiatric conditions, up until 2019 there has not been a gold standard social competencies assessment tool [19]. This means that the significance of the upper mentioned interventions can be enhanced by the reliability and sensitivity of the instrument used.

Metacognitive intervention targeting global cognitive function with focus on problem solving: In this single study [11], problem solving principles depending on metacognitive concepts were trained and administered and were to be investigated without a prior direction or direct outcome measure. For this cause and as it can be seen, 5 outcome measures were selected and used; Test of Nonverbal Intelligence-3 (TONI-3) which measured non-verbal abstract reasoning abilities, Metacomponential Interview (MI) which measured metacognition, Interpersonal Negotiation Strategies Interview (INSI) which measured problem-solving functional behaviour in social situations, Behavior Rating Inventory of Executive Function (BRIEF) which measured problem-solving functional behaviour in the home environment, and the Canadian Occupational Performance Measure (COPM) which measured self-perceived goal-directed functional behaviour individually. Up to the reviewer's knowledge, the outcome measures seem to be dispersed and not fully focusing on a single aspect of cognition as this is prevailed in the title of the study as "The effects of problem-solving skills training based on metacognitive principles for children with acquired brain injury attending mainstream schools". It yet showed significance on all levels of outcome measures as reported by the study [11,20-28]. Better links should be established between the outcome measures of this study and the functional goals of the training [29-34] (Figure 1).

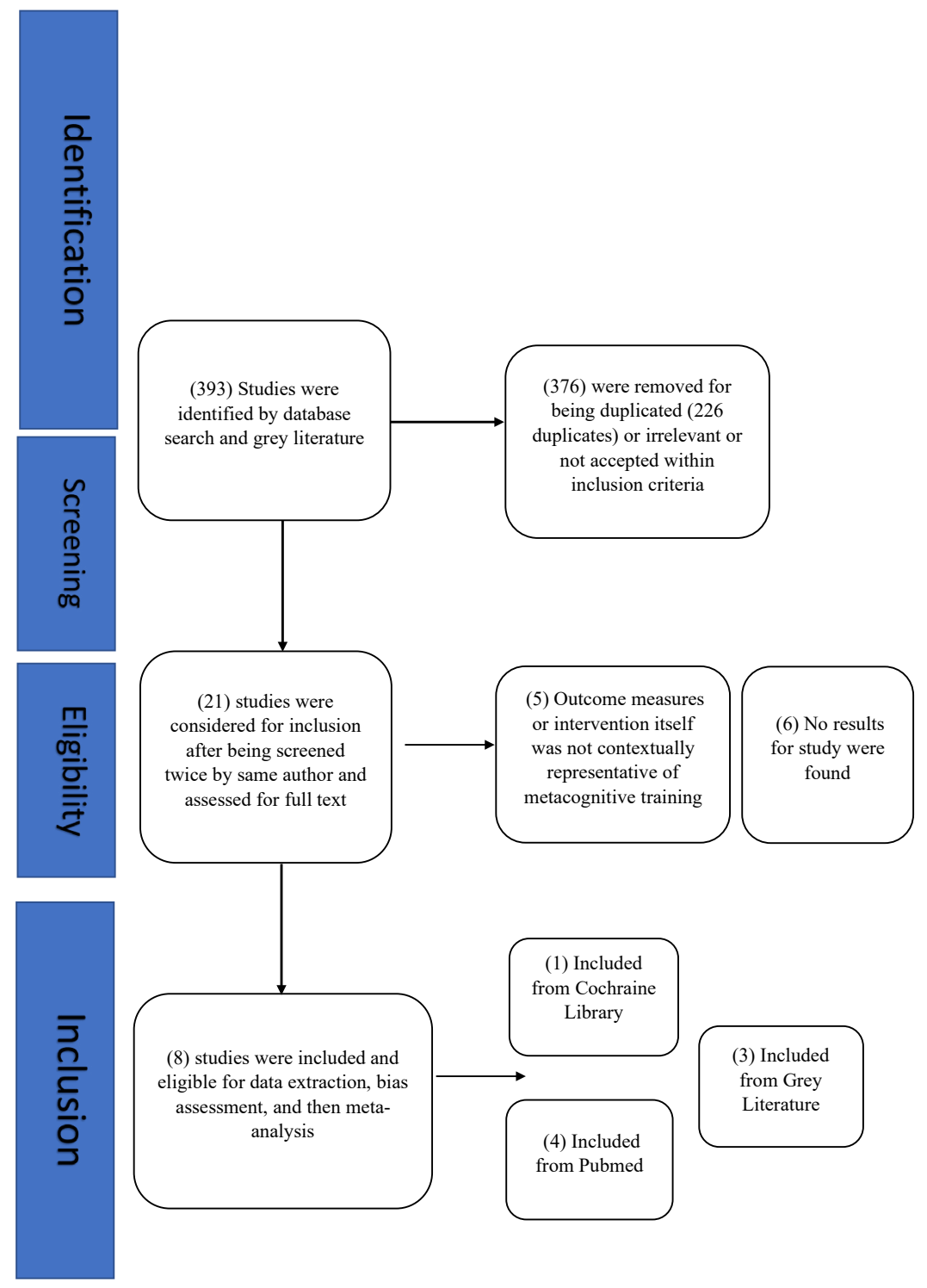

Figure 1. Study Flow Diagram 


\section{Conclusion}

If this review is to be successful, both researchers and clinicians should make use of it. On the literature level, we were able to detect how (MST) has been implemented and investigated on different cognitive levels. It might be the interest of other research domains to study whether metacognitive function can influence other impairments. Those impairments are not only cognitive since impression of self plays a vital role in its functionality as reported by Albert Bandura (who was the first to introduce the term "Self-efficacy"). People with higher self-efficacy report higher functionality and wellness of life. Upon the important discussions in neuropsychology currently is the macro versus micro understanding of neural networks. One of the causes of this controversy is the development of the instruments used to detect and read neural behavior. Another reason is the progress of psychotherapy and other rehabilitation techniques on the level of behavior altering and MRI detectable changes on the brain. This assists us in proposing better framework for our theories and application when it comes to understanding the brain. If clinicians are to benefit from this study, MST is not being introduced as a new concept but it is re-affirmed as a protocol for assessment and behavior for TBI patients more than simply an approach. Yet, the implementation of this approach must be better dissected. Literature has shown that metacognitive skills training is by definition distinct from other approaches which assess and target EF but metacognitive training sessions and modules are still relative and contrasting and have not yet evolved to be very different from executive function rehabilitation approaches - but this can be due to the wide umbrella of control that executive function has over other cognitive skills. This contrasting is not creativity yielding as much as it is uniqueness depriving. Despite metacognitive skills training not being an official protocol, it still stands out to be an essential part of neurorehabilitation and cognitive rehabilitation. Metacognitive training can help the patient gain insight and better enhance his error detection skills whether they were encompassed in his EF or not. From a clinical background, metacognitive assessment should be an initial phase added to the post hospitalization rehabilitation in centers equipping TBI patients with skills and generalized daily activity regimes. If it is to give two main recommendations; it is to insert metacognitive skills training in the official protocol of cognitive rehabilitation and other rehabilitation techniques and consider metacognition as an essential component to be assessed before rehabilitation. It is also good to mention that meta-topics are recently being re-explored from new backgrounds. If this review is to offer anything; then it should be "reconsideration" of our official protocols in rehabilitation.

\section{References}

1. Cornis-Pop M, Mashima PA, Roth CR, MacLennan DL, Picon LM, et al. (2012) Cognitive-communication rehabilitation for combat-related mild (TBI). Journal of Rehabilitation Research and Development 49: 11-31.

2. Article R, Zettin M, Galetto V (2016) Efficacy of The Metacognitive Training on Brain Injured Subjects. In EC Psychology and Psychiatry.

3. Dawson DR, Gaya A, Hunt A, Levine B, Lemsky C, et al. (2009) Using the Cognitive Orientation to Occupational Performance (CO-OP) with adults with executive dysfunction following (TBI). Can J Occup Ther 76: 115-127. [Crossref]

4. Finch E, Cornwell P, Copley A, Doig E, Fleming J (2017) Remediation of social communication impairments following (TBI) using metacognitive strategy intervention: a pilot study. Brain Injury 31: 1830-1839. [Crossref]

5. Franzen KM, Roberts MA, Schmits D, Verduyn W, Manshadi F (1996) Cognitive remediation in pediatric (TBI). Child Neuropsychology 2: 176-184.

6. Krasny-Pacini A, Limond J, Evans J, Hiebel J, Bendjelida K, et al. (2014) Contextsensitive Goal Management Training for everyday executive dysfunction in children after severe (TBI). J Head Trauma Rehabil 29: E49-E64. [Crossref]
7. Kurowski BG, Wade SL, Kirkwood MW, Brown TM, Stancin T, et al. (2014) Long-term benefits of an early online problem-solving intervention for executive dysfunction after (TBI) in children A randomized clinical trial. JAMA Pediatrics 168: 523-531. [Crossref]

8. Ownsworth T, Fleming J, Desbois J, Strong J, Kuipers P (2006) A metacognitive contextual intervention to enhance error awareness and functional outcome following (TBI): A single-case experimental design. J Int Neuropsychol Soc 12: 54-63. [Crossref]

9. Ownsworth T, Quinn H, Fleming J, Kendall M, Shum D (2010) Error self-regulation following (TBI): A single case study evaluation of metacognitive skills training and behavioural practice interventions. Neuropsychol Rehabil 20: 59-80. [Crossref]

10. Waid-Ebbs JK, Daly J, Wu SS, Berg WK, Bauer RM, et al. (2014) Response to Goal Management Training in Veterans with blast-related mild (TBI). Journal of Rehabilitation Research and Development 51: 1555-1566.

11. Chan DYK, Fong KNK (2011) The effects of problem-solving skills training based on metacognitive principles for children with acquired brain injury attending mainstream schools: A controlled clinical trial. Disabil Rehabil 33: 2023-2032. [Crossref]

12. Chung CSY, Pollock A, Campbell T, Durward BR, Hagen S (2013) Cognitive rehabilitation for executive dysfunction in adults with stroke or other adult non-progressive acquired brain damage. Cochrane Database Syst Rev 30: CD008391. [Crossref]

13. Logan DM, Hill KR, Larson MJ (2015) Cognitive control of conscious error awareness Error awareness and error positivity (Pe) amplitude in moderate-to-severe (TBI) (TBI). Frontiers in Human Neuroscience. [Crossref]

14. McAvinue L, O'Keeffe F, McMackin D, Robertson IH (2005) Impaired sustained attention and error awareness in (TBI): Implications for insight. Neuropsychological Rehabilitation 15: 569-587.

15. Barman A, Chatterjee A, Bhide R (2016) Cognitive impairment and rehabilitation strategies after (TBI). Indian J Psychol Med 38: 172-181. [Crossref]

16. Levine B, Schweizer TA, O'Connor C, Turner G, Gillingham S, et al. (2011) Rehabilitation of executive functioning in patients with frontal lobe brain damage with goal management training. Front Hum Neurosci pp. 1-9.

17. Kennedy MRT, Coelho C, Turkstra L, Ylvisaker M, Sohlberg MM, et al. (2008) Intervention for executive functions after (TBI): A systematic review, meta-analysis and clinical recommendations. Neuropsychol Rehabil 18: 257-299. [Crossref]

18. Perlovsky L, Sakai K L (2014) Language and cognition. Frontiers in Behavioral Neuroscience.

19. Genova HM, Haight A, Natsheh JY, DeLuca J, Lengenfelder J (2019) The relationship between social communication and social functioning in pediatric TBI: A pilot study. Front Neurol 10: 850. [Crossref]

20. Arciniegas DB, Held K, Wagner P (2002) Cognitive Impairment Following Traumatic Brain Injury. Curr Treat Options Neurol 4: 43-57. [Crossref]

21. Dockree PM, O'Connell RG, Robertson IH (2015) Connecting clinical and experimenta investigations of awareness in (TBI). Handbook of Clinical Neurology 128: 511-524.

22. Hamzah N, Narayanan V, Ramli N, Veeramuthu V, Tan LK, et al. (2018) A preliminary report on the effect of cognitive rehabilitation therapy in improving cognitive function of attention following mild (TBI): A randomised controlled trial. Annals of Physical and Rehabilitation Medicine 61: e41. [Crossref]

23. Harvey PD (2019) Domains of cognition and their assessment. Dialogues Clin Neurosci 21: 227-237. [Crossref]

24. Jain S, Iverson LM (2020) Glasgow Coma Scale. StatPearls. [Crossref]

25. Kabu S, Jaffer H, Petro M, Dudzinski D, Stewart D, et al. (2015) Blast-associated shock waves result in increased brain vascular leakage and elevated ROS levels in a rat model of (TBI). PLOS ONE 10: 5.

26. Kim JJ, Gean AD (2011) Imaging for the Diagnosis and Management of (TBI) Neurotherapeutics 8: 39-53. [Crossref]

27. Kintz S, Hibbs V, Henderson A, Andrews M, Wright HH (2018) Discourse-based treatment in mild (TBI). Journal of Communication Disorders 76: 47-59.

28. Krasny-Pacini A, Limond J, Evans J, Hiebel J, Bendjelida K, et al. (2015) Selfawareness assessment during cognitive rehabilitation in children with acquired brain injury: A feasibility study and proposed model of child anosognosia. Disability and Rehabilitation 37: 2092-2106.

29. Lebeer J (2016) Significance of the Feuerstein approach in neurocognitive rehabilitation. NeuroRehabilitation 39: 19-35. [Crossref]

30. Lippi D (2017) Case study of Berengario da Carpi and Lorenzo de' Medici. British Journal of Surgery 104: 1272. 
31. Mckee AC, Daneshvar DH (2015) The neuropathology of (TBI). Handbook of Clinical Neurology 27: 45-66

32. Orfei MD, Robinson RG, Bria P, Caltagirone C, Spalletta G (2008) Unawareness of illness in neuropsychiatric disorders: Phenomenological certainty versus etiopathogenic vagueness. Neuroscientist 14: 203-222.
33. Séguin M, Lahaie A, Matte-Gagné C, Beauchamp MH (2018) Ready! Set? Let's Train!: Feasibility of an intensive attention training program and its beneficial effect after childhood traumatic brain injury. Ann Phys Rehabil Med 61: 189-196. [Crossref]

34. https://www.mayoclinic.org/diseases-conditions/traumatic-brain-injury/symptomscauses/syc-20378557

Copyright: $(02020$ Makka SA. This is an open-access article distributed under the terms of the Creative Commons Attribution License, which permits unrestricted use, distribution, and reproduction in any medium, provided the original author and source are credited. 